\title{
MedienPädagogik
}

Zeitschrift für Theorie und Praxis der Medienbildung

\section{Sexting und sexuelle Grenzverletzungen an Schulen}

\author{
Lehrpersonen als Ansprechpersonen bei nicht-konsensuellen Praktiken \\ Christina Witz ${ }^{1}$ (D) \\ ${ }^{1}$ Europa-Universität Flensburg
}

\begin{abstract}
Zusammenfassung
Dieser Beitrag beleuchtet den schulischen Umgang mit Sexting-Veröffentlichungen aus der Perspektive von Schülerinnen und Schülern. Zunächst werden die Prävention sexueller Gewalt als Aufgabe von Schule (auch im digitalen Raum) und das Phänomen Sexting beleuchtet. Im empirischen Teil wird aufgezeigt, was Schülerinnen und Schüler daran hindert, mit Lehrpersonen in Kontakt zu treten, wenn ihre intimen Aufnahmen veröffentlicht wurden oder sie darum wissen, dass Sexts anderer Jugendlicher kursieren. Neben dem Umstand, dass Lehrpersonen häufig nichts vom missbräuchlichen Umgang mit intimen Bildern erfahren, verweisen die Jugendlichen auf Einstellungsfragen und Machtasymmetrien, jedoch auch auf adoleszente und institutionelle Abgrenzungen. Dabei thematisieren die Schülerinnen und Schüler Vertrauen im Lehrpersonen-Schülerinnen und Schüler-Verhältnis, eine Trennung in öffentliche und private Sphäre, den Aspekt beidseitiger Scham sowie die Zuschreibung der Verantwortung an die Betroffenen (Victim Blaming). Der Artikel kommt zu dem Schluss, dass es einer Abwendung vom Devianz- und Abstinenzdiskurs und hin zu einer Anerkennung einvernehmlichen jugendlichen Sextings als Form digitaler sexueller Kommunikation bedarf, um ein Veröffentlichungsgeschehen im schulischen Rahmen angemessen besprechbar zu machen und formuliert Implikationen für die schulische Praxis.
\end{abstract}

Sexting and Sexual Boundary Violations in Schools. Teachers as Contact Persons for Non-Consensual Practices

\begin{abstract}
This article looks at how schools deal with sexting from the perspective of students. First, the prevention of sexual violence as a task of schools (also in the digital space) and the phenomenon of sexting are examined. The empirical part shows what prevents students from contacting teachers when their intimate images are published or when they know that sexts of other young people are circulating. In addition to the fact that teachers often
\end{abstract}


do not find out about the misuse of intimate images, the young people point to attitudinal issues and power asymmetries, but also to adolescent and institutional boundaries. In this context, the students address trust in the teacher-pupil relationship, a separation between the public and private spheres, the aspect of shame on both sides and the attribution of responsibility to those affected (victim blaming). The article concludes that it is necessary to turn away from the discourse of deviance and abstinence towards an acknowledgement of consensual adolescent sexting as a form of digital sexual communication in order to make the act of publishing in a school setting appropriately discussable and formulates implications for school practice.

\section{1. $\quad$ Einleitung}

M1: [E]s spielt halt eine wichtige Rolle, (.) wie Lehrer dazu stehen (.) und welches Gefühl sie vor allem einem geben, weil, (2 Sek.) sie sind nun mal ähm immerhin trotzdem in einer höheren Position als jetzt die Mitschüler, ne? (.) Die können eher einem was sagen, als jetzt, (.) wie gesagt, die Mitschüler. Und wenn die dann halt so (3 Sek.) konservativ da gegenüberstehen, (.) ist das ziemlich schwer, mit denen halt zu reden. (4_S_innen_1, P:LP3, 23-29)

Das Internet eröffnet Jugendlichen wichtige Informations-, Kommunikations- und Interaktionsräume, auch im Themenfeld Sexualität. So finden sie online Inhalte zu sexualitätsbezogenen Fragen, gestalten erotische Beziehungen und experimentieren mit ihrer sexuellen und geschlechtlichen Identität (vgl. Vogelsang 2017, 342). Dabei stellt sich der digitale Sozialisationsraum in der adoleszenten Autonomiebewegung und zunehmenden Abgrenzung gegenüber Erwachsenen als ein weitestgehend von der (pädagogisch sie begleitenden) Erwachsenenwelt unbeeinflusster dar (vgl. Eichenberg und Auersperg 2018, 31). Mit dem Zugang zu digitalen Medien und Social Media eröffnen sich für Jugendliche somit sowohl neue Möglichkeiten der Selbstbestimmung und des Ausprobierens als auch onlinebezogener Risiken wie sexuellen Grenzverletzungen und Viktimisierungen, die den Blicken Erziehungsberechtigter sowie Pädagoginnen und Pädagogen weitestgehend entzogen sind und nur punktuell deren Aufmerksamkeit erregen. Eine dabei in den vergangenen Jahren besonders in den Fokus gerückte Form digitaler sexueller Interaktion ist die Praktik des Sexting, also des Austauschs sexuell andeutender bis expliziter Bilder (Sexts ${ }^{1}$ ) via Smartphone (vgl. Döring 2015, 16). Während konsensuelles Sexting mit positivem Erleben und

1 Alltagssprachlich werden verschiedene Begriffe (z. B. Nudes, Nacktbilder, sexy Pics, Dickpicks etc.) genutzt, die hier alle unter dem Begriff Sext gefasst sind. Dabei unterscheiden sich die Bilder (Foto bzw. Video) erheblich bezüglich des Grades der Nacktheit sowie dem gezeigten Körperausschnitt (Detail- bis Ganzkörperaufnahme, mit oder ohne sichtbare primäre oder sekundäre Geschlechtsmerkmale) und unterliegen bezüglich ihrer Interpretation des Grades an Intimität geschlechtlich unterschiedlichen Zuschreibungen (vgl. Witz 2021). 
Entwicklungsmöglichkeiten in Zusammenhang steht (vgl. Dekker, Koops, und Briken 2016; Naezer und Oosterhout 2020), können ungewollte Veröffentlichungen von Sexts als sexuelle Grenzverletzung verstanden werden und mit gravierenden Konsequenzen für die abgebildete Person einhergehen (vgl. Dekker, Koops, und Briken 2016; Van Ouytsel et al. 2016), wodurch die Frage nach kompetenter Unterstützung Betroffener aufgeworfen wird.

Obwohl regelmässig Veröffentlichungen von Sexts unter Schülerinnen und Schülern bekannt werden, ist der schulische Umgang damit bislang kaum erforscht (vgl. Böhm, Budde, und Dekker 2018). Dabei kommen Schulen neben der Vermittlung einer sexualitätsbezogenen Medienkompetenz (vgl. Vogelsang 2017) auch Aufgaben der Prävention und Intervention zu. Letzteres ist kürzlich mit der erneuten Aufforderung zum institutionellen Schutz vor sexueller Gewalt (vgl.«Bund und Länder gemeinsam gegen sexuelle Gewalt an Schulen» o. J.) bekräftigt worden. Dabei geht es, angewendet auf jugendlichen Bildertausch, einerseits darum, sextingbezogene Grenzverletzungen, Mobbing und Viktimisierung im Vorfeld entgegen zu wirken und andererseits im Fall von Veröffentlichungsgeschehen oder Erpressung mit intimen Bildern (Sextortion) ${ }^{2}$ sekundäre Viktimisierung, also zusätzliche negative Wirkungen für die Betroffenen durch fehlerhaftes Verhalten von Personen im Umfeld oder durch Institutionen der Hilfe (vgl. Schneider 2014), zu reduzieren. Für Fachkräfte im schulischen Rahmen bedeutet dies, in Veröffentlichungsgeschehen vertrauensvolle und kompetente Ansprechperson für Betroffene (sowie in anderer Form am Veröffentlichungsgeschehen beteiligte Schülerinnen und Schüler) zu sein und Zugang zu professioneller Hilfe zu bieten. Dabei ist wichtig zu verstehen, welche Faktoren Offenlegungen (Disclosure ${ }^{3}$ ) im Wege stehen und somit das Risiko weiterer Schädigung der Betroffenen fördern.

Dieser Beitrag möchte sich aus der bisher nicht beleuchteten Perspektive von Schülerinnen und Schülern der Frage nähern, was die Offenlegung nicht-konsensueller Umgangsweisen mit Sexts gegenüber Lehrpersonen behindert. Zunächst werden die Bedeutung von Schule bei der Prävention sexueller Gewalt und Erkenntnisse zur Rolle von Lehrpersonen bei der Offenlegung von Gewalterfahrungen vorgestellt (Abschnitt 2). Im Anschluss werden das Phänomen Sexting und nicht-konsensuelle Praktiken sowie die Frage der Unterstützung Betroffener anhand ausgewählter Forschungsergebnisse umrissen (Abschnitt 3). Im anschliessenden Abschnitt werden das Datenmaterial und das methodische Vorgehen beschrieben (Abschnitt 4), anhand dessen dann im empirischen Teil Hinderungsgründe für Offenlegungsgespräche mit Lehrpersonen herausgearbeitet und diskutiert werden (Abschnitt 5). Zum Abschluss werden die Ergebnisse zusammen gefasst und Implikationen für den schulischen Umgang mit Bild-Veröffentlichungen formuliert (Abschnitt 6).

2 Dabei können die abgebildeten Bilder zunächst freiwillig verschickt worden sein oder aber andere haben diese unrechtmässig in ihren Besitz gebracht.

3 Disclosure bezeichnet «das erstmalige und absichtsvolle Berichten über erlebte sexualisierte Gewalt oder anderes Unrechtserleben» (Oeffling, Winter, und Wolff 2018, 222). 


\section{Prävention und Intervention bei (digitaler) sexueller Gewalt als Aufgabe von Schule}

Schule, als zentralem Lern- und Begegnungsort von Kindern und Jugendlichen, wird im aktuellen bundesweiten Diskurs eine bedeutsame Stellung in der Prävention und Intervention zum Thema sexuelle Gewalt zugeschrieben. So setzt auch die Bundesinitiative «Schule gegen sexuelle Gewalt» des Unabhängigen Beauftragten für Fragen des sexuellen Kindesmissbrauchs (UBSKM) dabei an, Schulen bei der Entwicklung und Etablierung von Schutzkonzepten zu unterstützen (vgl. Glammeier 2019, 197). Dem Aspekt digitaler Medien wird dabei unter Anerkennung der Bedeutung heutiger medialer Sozialisation besondere Bedeutung beigemessen (vgl. «Sexuelle Gewalt mittels digitaler Medien - UBSKM» o. J.). Gleichzeitig zeigt eine aktuelle Metaanalyse des Forschungsstandes zu sexueller Gewalt und Schule auf, dass das Professionsverständnis von Lehrpersonen, inwieweit sie über rein didaktische Aufgaben der Prävention und Intervention hinaus aktives Handeln als Teil ihrer beruflichen Rolle sehen, wenig empirisch untersucht wurde und schulspezifisch unterschiedlich ausgeprägt ist (Christmann 2021). Auch verweisen Befunde aus Befragungen von Lehrpersonen auf grosse Wissenslücken sowie auf eine «ungünstige Handlungsorientierung» (Glammeier 2019, 206) für das Gelingen schulischer Prävention und Intervention. Zudem erleben Fachkräfte sexuelle Gewalt mittels digitaler Medien als besondere Herausforderung (vgl. Dekker, Koops, und Briken 2016: 3f). Darüber hinaus zeigt eine Untersuchung zu Offenlegungen sexueller Gewalterfahrung im schulischen Rahmen auf, dass Jugendliche sich vornehmlich an andere Jugendliche wenden und Erwachsene seltener als Ansprechpersonen nutzen, insbesondere, wenn sexuelle Übergriffe durch Gleichaltrige geschehen (vgl. Hofherr 2019, 146f). Wenden sich Jugendliche an Erwachsene, so sind dies vornehmlich Bezugspersonen wie Eltern, Lehrpersonen und selten nicht persönlich bekannte Vertreterinnen und Vertreter von Strafverfolgungsbehörden und Jugendhilfe (vgl. ebenda, 147). Zwar zeigen sich Beratungs- und Vertrauenslehrkräfte zunehmend verantwortlich für das Thema sexuelle Gewalt, jedoch sind sie - anders als z. B. Klassenlehrpersonen, zu denen Schülerinnen und Schüler langfristigen und engeren Kontakt haben - selten in Disclosureprozesse eingebunden (vgl. Christmann 2021). Während Schule als bedeutsames Feld zur Vermittlung sexualitätsbezogener Medienkompetenz adressiert wird (vgl. Vogelsang 2017) und sich für digital erlebte Gewalterfahrungen, die sich unter dem Begriff Cybermobbing fassen, zeigen lässt, dass Lehrpersonen aus Perspektive der Schülerinnen und Schüler kaum als Ansprechpersonen in online vermittelten Mobbingdynamiken gesehen werden (vgl. Schultze-Krumbholz et al. 2018a, 9), fehlt es bisher an Untersuchungen zu Ansprechpersonen für Schülerinnen und Schüler in Veröffentlichungsgeschehen rund um sexuell intime Aufnahmen und spezifisch der Frage, warum sich Schülerinnen und Schüler gegenüber Lehrpersonen selten anvertrauen. 


\section{Jugendliches Sexting, nicht-konsensuelle Praktiken und Hilfe für die Betroffenen}

Sexting, verstanden als digital vermittelter konsensueller Austausch selbstgenerierter sexuell andeutender bis expliziter Bilder (vgl. Döring 2015, 16), ist eine vornehmlich von Erwachsenen praktizierte Form intimer Kommunikation, die auch unter Jugendlichen in den letzten Jahren zugenommen hat und mit dem Alter - und damit insgesamt wachsenden sexuellen Erfahrung - der Jugendlichen steigt (vgl. Madigan et al. 2018). So werden in einer aktuellen Übersichtsstudie für das Senden und Empfangen von Sexts unter 12- bis 17-Jährigen durchschnittliche Prävalenzen von 14,8 \% bzw. $27,4 \%$ benannt (vgl. ebenda). Lange Zeit herrschte in der wissenschaftlichen und pädagogischen Bearbeitung jugendlichen Sextings ein Abstinenz- und Devianzdiskurs vor, der sich erst in jüngerer Zeit in Richtung eines Normalitätsdiskurses wandelt (vgl. Döring 2015), nach dem jugendliches Sexting als zeitgemässe sexuelle Handlungsfähigkeit (agency) sowie digital vermittelte intime Erkundungs- und Ausdrucksmöglichkeit gefasst wird (vgl. Symons et al. 2018; Naezer und Oosterhout 2020). Da der Austausch von Sexts je nach Länderrecht unterschiedlichen Altersbestimmungen unterliegt und zum Teil erst mit Erlangung der Volljährigkeit legal wird, werden zunehmend Forderungen formuliert, gesetzliche Bestimmungen mit Berücksichtigung digitaler Entwicklungen und dem online-Verhalten von Jugendlichen zu reformieren (vgl. Strasburger et al. 2019). Nach deutschem Recht ist einvernehmliches Sexting hingegen auch unter Berücksichtigung des §184c, der den gesetzlichen Rahmen zu Verbreitung, Erwerb und Besitz jugendpornographischer Inhalte fasst, auf privater Ebene unter Jugendlichen erlaubt. Kommt es hingegen zu sexuellen Grenzverletzung in Form von nicht-konsensuellen Veröffentlichung intimer Aufnahmen, erfüllt dies unter Umständen gleich mehrere Straftatbestände für Veröffentlichende und Weiterleitende ${ }^{4}$. Dabei zeigt sich, dass es ihnen häufig nicht an Kenntnis der Rechtslage mangelt. Stattdessen leitet ein Grossteil der Jugendlichen auch dann Bilder ohne das Einverständnis der abgebildeten Person weiter, wenn sie um das Recht am eigenen Bild wissen (vgl. Vogelsang 2017). Vielfach wird darauf hingedeutet, dass Geschlechterdimensionen eine bedeutsame Rolle bei nicht-konsensuellen Praktiken spielen. Neben dem Aspekt, dass geschlechterstereotype Vorstellungen von Sexualität jugendliche Orientierungen bezüglich sexuell interpretierbarer Selbstdarstellungen prägen (vgl. Naezer und Oosterhout 2020; Witz 2021), erfahren insbesondere sextende Mädchen soziale Stigmatisierung sowie eine Schuldzuweisung in Veröffentlichungsvorgängen (Döring 2012; Naezer und Oosterhout 2020; Budde et al. 2019). In Bezug auf Betroffene zeigen Johnson et al. auf, dass Jugendliche wenig Hilfemöglichkeit für diejenigen sehen, deren Bilder veröffentlicht wurden (vgl. Johnson et al. 2018), und auch in der Studie von Vogelsang schätzen über 30 Prozent der befragten

4 Der nicht-einvernehmlichen Umgang mit Sexts stellt ein häufiges Phänomen unter Jugendlichen dar. Zur Ausdifferenzierung anderer Formen nicht-konsensueller Praktiken beim Sexting vgl. Döring 2015, 29ff. 
Jugendlichen die Situation für die Abgebildeten weitergeleiteter Sexts als «(eher) hilf- und machtlos» (Vogelsang 2017, 294) ein. Gleichzeitig beschreiben Studien die häufig fehlende Unterstützung Betroffener mit Aspekten von Abwertung (u. a. Slutshaming) und Schuldumkehr (Victim Blaming) seitens Mitschülerinnen und Mitschülern, Eltern sowie Lehrpersonen (Naezer und Oosterhout 2020). ${ }^{5}$

\section{Datenmaterial und methodisches Vorgehen}

Das Datenmaterial für diesen Beitrag bilden zwölf geschlechtshomogene Gruppendiskussionen mit Schülerinnen und Schülern unterschiedlicher Schulsysteme der Klassenstufen 10 und 11, die im Rahmen der durch das Bundesministerium für Bildung und Forschung (BMBF) geförderte Studie SaferSexting - Sexuelle Grenzverletzungen mittels digitaler Medien an Schulen geführt wurden, um Zusammenhänge zwischen sexueller Gewalt, digitalen Medien und schulischem Kontext, zu erforschen. ${ }^{6}$ Die leitfadengestützten und erzählgenerierend geführten Gruppendiskussionen mit den Schülerinnen und Schülern wurden vollständig transkribiert und in Anlehnung an die Dokumentarische Methode nach Bohnsack ausgewertet. Dabei wird in der Dokumentarischen Methode zwischen explizitem und implizitem, also dem wörtlich kommunizierbaren und einem atheoretischen Wissen differenziert, wobei sich letzteres sowohl in sozialen Handlungen als auch in narrativen Interaktionsprozessen der Akteurinnen und Akteure ausdrücken kann. Auf diese Weise ist es möglich, kollektive Orientierungen im Sinne gemeinsam geteilter und diskursivierbarer Wissensbestände zu untersuchen, die in der Dokumentarischen Methode als handlungsleitend für die soziale Praxis verstanden werden (vgl. u. a. Bohnsack 2013, 250).

Die hier zusammen gestellten Passagen werden anhand des Analyseschrittes auf der Ebene einer reflektierenden Interpretation (Przyborski 2004, 55) präsentiert und verfolgen einen Nebenstrang zur Hauptauswertung der Untersuchung, in der kollektive Orientierungen von Schülerinnen und Schülern in Bezug auf Sexting und Bildveröffentlichungen untersucht werden. So nimmt dieser Beitrag aus Perspektive von Jugendlichen Aspekte schulischer Interventionshemmnisse bei der Frage der Offenlegung von grenzverletzenden Vorkommnissen rund um Sexting in den Blick. Die Auswahl der Passagen erfolgte dabei entlang der für die dokumentarischen Methode als zentral beschreiben Kriterien, die neben einem thematischen Zugang formale Aspekte umfassen. Da die hier präsentierte Teilauswertung jedoch nicht auf die

5 Die Erzählungen über Veröffentlichungsszenarien in den Gruppendiskussionen mit Lehrpersonen, Schülerinnen und Schülern sowie die quantitativen Befragungen von Schulleitungen im Rahmen des Forschungsprojektes SaferSexting verweisen zudem darauf, dass wesentlich häufiger diejenigen, deren Bilder weiter geleitet wurden, die Schule wechseln als diejenigen, die die Bilder weiter geleitet haben.

6 Das diesem Bericht zugrundeliegende Vorhaben wurde mit Mitteln des Ministerium für Bildung und Forschung unter dem Förderkennzeichen FKZ 01SR1708A gefördert. Die Verantwortung für den Inhalt dieser Veröffentlichung liegt bei der Autorin. 
Beschreibung fallübergreifender Typen im Sinne der dokumentarischen Typenbildung abzielt (vgl. Nentwig-Gesemann 2013), sondern Themen sowohl anhand mehrfacher Benennung als auch entlang von Einzelfällen herausarbeitet, wird hier der thematisch orientierte Zugang besonders gewichtet. Darüber hinaus kommen bei der Auswahl der Passagen auch die formalen Kriterien der «interaktiven Dichte» sowie der «metaphorischen Dichte» zum Tragen, die im Sinne von «Fokussierungsmetaphern» auf einen gemeinsamen Erfahrungshorizont verweisen. Unter ersterer wird das gemeinsame, sich ins Wort fallende oder auch gegenseitig ergänzende Sprechen verstanden, während zweite eine bildhafte Erzählweise bezeichnet (vgl. Bohnsack 2013, 250).

Diese auch am Einzelfall orientierte Herangehensweise ist für die Fragen schulischer Intervention bei sexueller Gewalt in Bezug auf den Umgang mit Sexts insofern relevant, als dass die Perspektive von Schülerinnen und Schülern bisher kaum beschrieben wurde und auch die Sicht von Betroffenen bei (angedrohter) Veröffentlichung aufschlussreich für die Verbesserung opferorientierter Hilfe bedeutsam ist. Somit lassen sich auch aus dem Einzelfall wertvolle Erkenntnisse für Prävention und Intervention ableiten.

\section{Lehrpersonen als Ansprechpersonen und Hinderungsgründe bei Veröffentlichungsgeschehen}

In den Gruppendiskussionen mit den Schülerinnen und Schülern wurde zum Teil selbstläufig, zum Teil auf immanente Nachfrage durch die Interviewerinnen und Interviewer (z. B. «Wie ist die Schule damit umgegangen?») auf Personen im schulischen Rahmen Bezug genommen. Dabei wurden vor allem Lehrpersonen, häufig Klassen- oder Fachlehrkräfte mit kontinuierlichem Bezug zu den Schülerinnen und Schülern, in den Erzählungen der Jugendlichen benannt, während Vertrauenslehrkräfte oder sozialpädagogischen Fachkräften zum Teil erwähnt wurden, jedoch häufig als zwar bekannte jedoch kaum in die schulische Praxis der Jugendlichen eingebundene erschienen. Im Folgenden werden anhand ausgewählter Passagen Aspekte heraus gearbeitet, die aus Perspektive der Schülerinnen und Schüler eine Offenlegung nicht-konsensueller Praktiken mit Sexts im schulischen Rahmen behindern. Dabei geht es neben der Frage grundlegenden Vertrauens (5.1) um generationale und institutionelle Grenzen (5.2), den Aspekt beidseitiger Scham (5.3) sowie Victim Blaming und Slutshaming in Bezug auf Betroffene der Veröffentlichungen (5.4). Im Anschluss werden die Ergebnisse diskutiert (5.5). 


\subsection{Vertrauen im Verhältnis von Schülerinnen und Schülern und Lehrpersonen}

In der folgenden Passage diskutieren Schülerinnen, was Lehrpersonen aus der Schülerinnen- und Schülerschaft mitbekommen und inwiefern diese für sie Vertrauenspersonen sind. Dabei konstatieren sie einen qualitativen Unterschied in der grundsätzlichen Besprechbarkeit privater Themen, der auf eine erschwerte Thematisierung sexueller Grenzverletzung durch nicht-konsensuelle Praktiken mit Sexts verweist.

M3: Ich glaube, die Lehrer haben es noch gar nicht so richtig mitbekommen.

M2: //Nein//.

M3: //Also,// die Schüler reden jetzt nicht so viel mit den Lehrern, weil, es sind irgendwie die Lehrer. Die geben die Noten. Und das sind für uns, also für $\mathrm{MICH}$ auch keine Vertrauenspersonen so.

M4: Mh, doch. Also-, //(schnaubendes Lächeln)//

M4: //fin-, ja i-,// also,

M3: //Ja.//

M4: //ich// habe doch schon, ähm, Vertrauen in meine Klassenlehrerin, zum Beispiel. Mit der habe ich auch viel geredet so allgemein private Sachen. Ähm, (.) da gibt-. Man kann sich halt schon öffnen, aber, es ist halt-. Nicht jeder ist halt dann so wie ich und geht dann auch zu Lehrern hin irgendwie. (.) Ähm, und, (2 Sek.) aber, also, mit den Lehrern wird halt schon viel kommuniziert. Und den Lehrern fällt, finde ich, auch ziemlich viel auf schon.

M3: Ja, aber es ist halt auch so, wenn der Schüler dann mit dem Lehrer nicht sprechen will, dann kann der Lehrer da auch nicht mehr viel machen und so. Und das, denke ich, ist auch häufig der Fall dann, bei //solchen// Sachen.

M4: $/ / \mathrm{Ja} / /$

(4_S_innen_2, P:LP1, 24-47)

M3 konstatiert mit Zustimmung von M2, dass Lehrpersonen das Kursieren von Bildern bisher nicht mitbekommen und elaboriert, dass Schülerinnen und Schüler sich nicht an Lehrpersonen wenden, da diese «irgendwie die Lehrer» sind, deren Aufgabe darin besteht, schulische Leistung zu bewerten («Die geben die Noten»). M3 schliesst Lehrpersonen als Vertrauenspersonen zunächst allgemein für Schülerinnen und Schüler («uns») aus, schränkt dies dann jedoch mit einer Betonung auf ihre Person ein. M4 setzt an und sucht nach Worten, um dann zu differenzieren, dass für sie persönlich ihre Klassenlehrerin eine Vertrauensperson darstellt, mit der sie auch viel über «so allgemein private Sachen» gesprochen hat. Es besteht die Möglichkeit sich zu öffnen und, wie sie, auf Lehrpersonen zuzugehen, was jedoch nicht für alle passend ist. Lehrpersonen wird zugestanden, viel mit Schülerinnen und Schülern zu kommunizieren und Probleme wahrzunehmen, da «ihnen ziemlich viel auffällt». 
M3 stimmt zu und differenziert dann weiter aus, dass Lehrpersonen jedoch nicht viel machen können, wenn Schülerinnen und Schüler nicht mit ihnen reden wollen. Sie schlussfolgert, dass dies in Bezug auf das Thema Sexting und Veröffentlichungen («solchen Sachen») häufig der Fall ist, was M4 abschliessend validiert.

Die Schülerinnen dieser Passage teilen die Orientierung, dass es individuell unterschiedlich ist, ob Schülerinnen und Schüler persönliche Vertrauensverhältnisse zu Lehrpersonen haben. So kann das System schulischer Bewertung einem Vertrauensverhältnis generell entgegen stehen. Hingegen ist es einzelnen möglich, aktiv Kontakt zu ausgewählten Lehrpersonen zu suchen, um persönliche Belange zu besprechen. Auch wenn Lehrpersonen von sich aus aufmerksam gegenüber den Belangen von Schülerinnen und Schülern sind, stellen der nicht-konsensuelle Umgang mit Sexts eine Besonderheit dar. Zudem erscheinen Lehrpersonen in ihren Möglichkeiten beschränkt, wenn Schülerinnen und Schüler das Gespräch verweigern, was im Falle nicht-konsensuellen Verschickens intimer Aufnahmen als hoch eingeschätzt wird. Indem die Schülerinnen «so allgemeine private Sachen» von «solchen Sachen» unterscheiden, kennzeichnen sie unterschiedliche Grade der Besprechbarkeit und zeigen auf, dass Belange um Sexting und Veröffentlichungsgeschehen eine Kommunikation mit Lehrpersonen neben der prinzipiellen Frage des Vertrauens zusätzlich erschweren.

\subsection{Zwischen Öffentlich und Privat - Zum Aspekt der Sphärentrennung entlang generationaler und institutioneller Grenzen}

Von den Schülern werden in der folgenden Passage sowohl die Grenze zwischen den Generationen als auch die Trennung entlang institutionsbezogener Positionen (Lehrpersonen vs. Schülerinnen und Schüler) als Hinderungsgründe für Aufdeckungen im schulischen Rahmen benannt und entlang dem Verhältnis von Öffentlichem und Privatem verhandelt.

J3: Ich habe halt das Gefühl so, solche Sachen-. Wir reden untereinander darüber, aber irgendwie trotzdem versuchen alle es so noch vor den Lehrern eher fernzuhalten.

J2: //Genau, weil es-//

J3: //Das ist so, keiner// geht zum Lehrer, weil keiner hat Bock dann, dass der Lehrer mit einem darüber redet.

J2: Genau. Weil es ist ja auch irgendwie trotzdem noch eine intime Sache und ich glaube //alle//

J4: //Ja.//

J3: Leute wissen auch, dass es was Intimes ist und wenn das dann so über die Lehrer geht, dann wird das ja zu was Offiziellem. Und //dadurch ist es glaube ich auch// 
J3: //Und plötzlich weiß die ganze Schule davon.//

J2: $\quad \mathrm{NOCH}$ unangenehmer und ich glaube für die anderen Beteiligten ist es dann auch wieder unangenehm und deshalb sprechen die es auch nicht an, weil (.) das ja schon irgendwie so ein intimer Einschnitt ins Privatleben ist so damit-, mit Erwachsenen auch so zu sprechen glaube ich. Weil was unter Kindern ist, bleibt unter Kindern. //Ist halt meistens so.//

J3: //Ist so.//

$\mathrm{J} 1: \quad / / \mathrm{Ja} . / /$

(6_S, P:5, 26-46)

Die Jungen berichten, dass Schülerinnen und Schüler untereinander über verschickte oder kursierende Sexts sprechen, während Lehrpersonen davon nichts erfahren sollen («vor den Lehrern eher fernzuhalten»). Es dokumentiert sich eine zweifache Sphärendifferenz, in der die Schülerinnen und Schüler versuchen, Distanz zur Erwachsenenwelt sowie zur Institution Schule aufrecht zu erhalten. Als Hinderungsgrund, Erwachsene im schulischen Rahmen anzusprechen, benennen die Schüler zunächst individuelle Unlust («keiner hat Bock»), dass Lehrpersonen «mit einem darüber rede[n]». Denn obwohl unter Schülerinnen und Schülern bekannt, wird durch das Gespräch mit einer Lehrperson eine «irgendwie trotzdem noch [...] intime Sache» «zu was Offiziellem», von dem dann die gesamte Institution («die ganze Schule») erfährt. Die Schüler benennen zudem den Aspekt der Scham, der sich durch eine Aufdeckung für die abgebildete Person noch erhöht («NOCH unangenehmer»), aber auch auf der Ebene aller anderen involvierten Personen eine Rolle spielt. Die Schüler wiederholen die Trennung der jugendlichen Sphäre von der Erwachsenenwelt, indem sie ein Gespräch mit Erwachsenen über Sexting und Veröffentlichungsgeschehen als «intime[n] Einschnitt ins Privatleben» benennen, und sich selbst eher der Sphäre der Kindheit als des Erwachsenenalters zugehörig markieren («Was unter Kindern ist, bleibt unter Kindern»).

Es dokumentiert sich in dieser Passage die kollektive Orientierung einer doppelten Sphärentrennung, in der die adoleszente private Sphäre Jugendlicher von der öffentlichen schulischen Sphäre erwachsener Akteurinnen und Akteure getrennt ist. Als Veröffentlichung gilt nicht das Kursieren von Bildern oder diesbezügliche Kommunikation unter Jugendlichen, sondern serst die Preisgabe im Rahmen der schulischen Institution und gegenüber der Erwachsenenwelt. Sexting - aber auch damit im Zusammenhang stehenden Grenzverletzungen unter Jugendlichen - wird dem jugendkulturell-Privaten zugeordnet, welches erst bei Aufdeckung gegenüber Erwachsen zu einer offiziellen Angelegenheit wird, wodurch eine Potenzierung der Scham für alle Beteiligten droht. Gleichzeitig schreiben die Schüler der Gruppendiskussion in der Trennung der Sphären die Grenzverletzung unter Jugendlichen fort, indem sie als (zumindest wissend um ein Veröffentlichungsgeschehen) Involvierte 
der unangenehmen Situation eines Gespräches mit Lehrpersonen ausweichen und damit Interventionsmöglichkeiten durch Erwachsene und Hilfe für die betroffene Person erschweren.

\subsection{Beidseitige Scham als Hinderungsgrund mit Lehrpersonen zu sprechen}

Die Schülerinnen der folgenden Passage diskutieren, wie Lehrpersonen reagieren würden, wenn betroffene Schülerinnen und Schüler sich an sie wenden und arbeiten gemeinsam den Aspekt der beidseitigen Scham als Hinderungsgrund heraus. M2 vermutet zunächst, dass Lehrpersonen vieles, was Schülerinnen und Schüler in der Klasse oder im Jahrgang untereinander wissen, nicht mitbekommen, da es «über die Medien passiert» und sie mit Lehrpersonen nicht «über Medien in Kontakt» sind und führt dann weiter aus:

M2: [...] Aber, ich meine, ich weiß jetzt gar nicht, wie Lehrer darauf reagieren würden, ob auch wirklich Leute da (.) dann auf den Lehrer zugehen und das sagen und dass da was vorgefallen ist. Weil, (.) ich meine, (.) irgendwie gehört das ja dann doch auch mit Schamgefühl dazu, weil man das vielleicht auch nicht möchte, dass es gerade der Lehrer mitbekommt. Ich meine, wenn das schon die Mitschüler wissen, dann ist das schon schlimm genug. Und dass das dann vielleicht noch der Lehrer-, oder dann besprochen wird, wo auch immer in der Klasse, oder nur mit dem Lehrer will halt vielleicht nicht jeder.

M3: Wobei, ich glaube, wenn man zum Lehrer geht, würde der einem schon echt helfen. Und-

M4: Ja.

M3: auch, wenn es unangenehm ist. Aber, die würden jetzt nicht sagen: Nee, musst du jetzt selber sehen, wie du zurechtkommst. (.) Also-.

M4: Wobei ich auch sagen muss, also, Lehrer sind ja auch irgendwie Menschen. Also, ist ja einfach so. Und die haben ja auch ihre Gefühle irgendwie. Und bei dem einen Lehrer sieht man es halt mehr und bei dem anderen halt eher weniger. Und (.) bei einigen oder bei (.), doch bei einigen, habe ICH persönlich schon das Gefühl, dass es denen UNANGENEHM wäre, wenn man darüber spricht. Also, als wir das Thema Sexualkunde hatten, hatte ich mit der, ähm, mit der Lehrkraft halt nicht das Gefühl, dass ihr das hundertprozentig, ähm, also, dass sie da sachlich sozusagen darüber sprechen kann, so, sondern, dass das für sie auch unangenehm ist, darüber zu sprechen. (.) Was an sich natürlich nicht schlimm ist. (.) Aber, was das Ganze halt schwieriger macht, weil es natürlich dann für un-, für einen selber dann so ein bisschen Unruhe macht, dass sogar eine erwachsene Person sozusagen damit nicht (.) nicht klar, sozusagen, kommt irgendwie.

(4-S_innen_2, LP:L4, 28-48) 
M2 erklärt, dass ihr nicht klar ist, wie Lehrpersonen auf die Offenlegung eines nicht-konsensuellen Sexting-Geschehens reagieren und ob Schülerinnen und Schüler sich «wirklich» mit der Bitte um Hilfe an sie wenden. Als Begründung nennt sie «Schamgefühl» und vermutet, dass viele Schülerinnen und Schüler gerade gegenüber Lehrpersonen nicht wünschen, dass diese erfahren, was vorgefallen ist. Für die betroffenen Personen ist es bereits beschämend («schon schlimm genug»), wenn die Mitschülerinnen und Mitschüler darum wissen, so dass jedes weitere Gespräch mit Lehrpersonen oder die Thematisierung in der Klasse vermutlich seitens der Betroffenen meist nicht erwünscht ist, da dies das Potenzial erneuter Beschämung in sich birgt. M3 wendet mit Zustimmung von M4 ein, dass Lehrpersonen einem «schon echt» helfen würden, wenn eine Schülerin oder in Schüler an sie herantritt, auch wenn die Situation «unangenehm» ist. Nach einer kurzen Pause setzt M4 an und differenziert den Aspekt der Scham im Verhältnis von Schülerinnen und Schülern und Lehrpersonen weiter aus. Sie konstatiert zunächst, dass «Lehrer [...] ja auch irgendwie Menschen» sind, die «ihre Gefühle» haben, die für die Schülerinnen und Schüler unterschiedlich sichtbar sind. Sie vermutet, dass es einigen von ihnen «UNANGENEHM» wäre, über nicht-konsensuelle Sexting-Praktiken mit Schülerinnen und Schülern zu sprechen und rekurriert auf ihre Erfahrung im Sexualkundeunterricht. M4 hatte nicht das Gefühl, dass die Lehrperson «sachlich» über sexuelle Themen («darüber») sprechen konnte. Dies legt sie der Lehrperson nicht als Fehler aus («nicht schlimm ist»). Es erschwert jedoch die Situation für Schülerinnen und Schüler («das Ganze halt schwieriger macht»), sich in einer unangenehmen Situation an Erwachsene zu wenden, da es verunsichernd ist («so ein bisschen Unruhe macht»), dass sogar ein erwachsenes Gegenüber die Situation nicht halten kann («nicht klar [...] kommt»). Somit überwiegt in der Abwägung der Schutz der Lehrperson vor einer Konfrontation mit für diese unangenehmen Themen das eigene Hilfebedürfnis.

Die Schülerinnen teilen die Orientierung, dass Scham für die Offenlegung eines nicht-konsensuellen Umgangs mit Sexts eine bedeutsame Rolle spielt. Ähnlich der Passage zuvor, beschreiben auch sie zunächst die Offenlegung gegenüber Lehrpersonen und die Thematisierung im grösseren schulischen Rahmen für die betroffene Person als schamverstärkend. Obwohl sie Lehrpersonen eine grundsätzliche Bereitschaft zu helfen zusprechen, sehen sie darüber hinaus deren generelle Unsicherheit im Umgang mit sexuellen Themen als unterstützungshinderlich. Die spürbare Scham Erwachsener erschwert es Schülerinnen und Schülern, sich mit einem unangenehmen, schambesetzten Thema an sie zu wenden, wenn sie nicht einschätzen können, ob diese mit dem Anvertrauten hilfreich und unterstützend umgehen kann. 


\subsection{Zuschreibung der Verantwortung an die abgebildete Person (Victim Blaming und Slutshaming)}

Die Schülerinnen der folgenden Passage erarbeiten gemeinsam eine sexting-ablehende Grundhaltung von Lehrpersonen, die zu einer Zuschreibung der Verantwortung an die abgebildete Person führt (Victim Blaming) und auf Mädchen bezogene geschlechtliche Abwertung beinhaltet (Slutshaming). M4 berichtet dabei aus der Perspektive einer Betroffenen, deren innerhalb einer Beziehung verschickte SextingAufnahme von ihrem Partner veröffentlicht wurde. Die hohe Reichweite der Verbreitung ihres Sexts ging mit massivem Mobbing und Slutshaming, also der Verurteilung eines sexuell aktiv in Erscheinung tretenden Mädchens als "Schlampe», in und ausserhalb der Schule einher und führte zu umfassender psychischer und sozialer Belastung der Schülerin.

M4: [...] Ich glaube, weswegen auch ganz viele nicht mit Lehrerin, äh Lehrern reden ist, weil viele Lehrer so eine Abneigung dagegen haben und (.) Dich dann auch anders sehen. Also ich hatte das Problem, (.) ich habe mit ALLEN Lehrern gesprochen, die wir hatten.[...] Ich habe das erzählt (.) und ich habe nur von einem Lehrer eine Rückmeldung bekommen, dass er was dagegen tun wird, dass das so nicht weitergehen kann. Die meisten haben gar nichts gesagt, halt diese normale Reaktion einfach, ja, wir machen das. Hier, da. Haben aber nie irgendwas gemacht. Ich habe teilweise auch Reaktionen bekommen von wegen, du bist ja selber Schuld. Du trägst ab und zu ein enges Oberteil. Du bist ja selber Schuld, dass sie dich als Hure bezeichnen. (2 Sek.) Wo ich mir denke, (.) nur weil ich eine Frau bin und mal ein enges Oberteil trage, bin ich dann gleich eine Nutte?

M1: Mhh (stöhndend)

M2: Ja, (Lächeln) vor allem das finde ich auch immer so geil, so, ja, ähm (.) selbst Schuld, dass du vergewaltigt worden bist bei so einem //kurzen//

M4: //Ja.//

M2: Kleid, irgendwie. Dann denke ich mir, seit wann sagen meine Klamotten JA?, irgendwie.

M4: $/ / \mathrm{Ja} / /$

M2: //Seit wann// reden meine Klamotten für mich, irgendwie.

(4_S_innen_1, P:4, 25-47)

M4 vermutet, dass sich Jugendliche im Rahmen von Schule häufig nicht an Erwachsene wenden, da viele Lehrpersonen Sexting generell ablehnend gegenüber stehen («so eine Abneigung dagegen haben») und Schülerinnen und Schüler mit dem Wissen um deren Sexting-Aktivität verurteilen («Dich dann auch anders sehen»). Sie erzählt in einem gemeinsamen Treffen mit allen sie unterrichtenden Lehrpersonen, was ihr passiert ist, erhält jedoch nur von einem Lehrer die Zusage aktiver 
Unterstützung. In der Ausführlichkeit ihrer Schilderung dokumentiert sich ein Gefühl der Ohnmacht, vom System Schule mit der Situation allein gelassen worden zu sein. Darüber hinaus schreiben ihr Lehrpersonen die Verantwortung für die ihr widerfahrene Abwertung als «Hure» zu und begründen dies mit ihrem Kleidungsstil, dem gelegentlichen Tragen eines engen Oberteils, wodurch sich die Lehrpersonen in das Slutshaming der Schülerinnen und Schüler einreihen. Rhetorisch stellt M4 die Frage, ob sie gleich eine Nutte sei, wenn sie als Frau mal ein enges Oberteil trägt und rekurriert dabei auf eine Dramatisierung, der sich unter Kleidung abzeichnenden weiblichen Brust. ${ }^{7}$ Gemeinsam elaborieren die Schülerinnen daraufhin die Unangemessenheit der Verantwortungszuschreibung (Victim Blaming). Diese dokumentiert sich zunächst in der direkten Antwort des Stöhnens («Mhh») sowie der nachfolgenden leicht lachend gesprochenen ironischen Einleitung («finde ich immer so geil, so, ja»), die dann in der Analogie der zur Metapher gewordenen Schuldumkehr bei Vergewaltigungen mündet: eine Frau, die sich in einen kurzen Rock kleidet, trägt (Mit-)Schuld, wenn sie vergewaltigt wird. Indem die Schülerinnen die Zuschreibung der Verantwortung für das M4 widerfahrene Slutshaming mit der Schuldumkehr bei Vergewaltigungen parallelisieren, übersetzen sie den Kampf der Frauenbewegung um die Anerkennung einer sexuellen Gewalthandlung als solche in die digitalisierte Welt. Denn während für offline ausgeübte sexuelle Gewalt ein breiter gesellschaftlicher und juristischer Konsens darüber besteht, dass die Gewalthandlung nicht mit dem Tragen bestimmter Kleidung des Opfers begründet werden darf, monieren die Schülerinnen und Schüler eine fehlende Übertragung seitens Erwachsener in die digitale Welt. Zugleich unterstreichen sie den Gewaltaspekt einer Sexting-Veröffentlichung und skandalisieren die verweigerte Unterstützung durch das Lehrpersonal der Schule.

\subsection{Diskussion der Ergebnisse}

Anhand der vier interpretierten Passagen wurden verschiedene Aspekte heraus gearbeitet, die für Schülerinnen und Schüler - aus der Perspektive von Betroffenen aber auch aus der Perspektive derjenigen, die von nicht-konsensuelle Praktiken im Umgang mit Sexts erfahren - eine Offenlegung (Disclosure) behindern. So können Lehrpersonen vereinzelt Vertrauenspersonen sein, mit denen auch private Dinge besprochen werden. Berühren die Anliegen der Schülerinnen und Schüler jedoch sexualitätsbezogene Themen und den Umgang mit intimem Bildmaterial, beschreiben sie eine Einschränkung der Kommunikationsmöglichkeit. In der Forschung zu (nicht-spezifisch digital vermittelter) erlebter sexueller Gewalt wird darauf hingewiesen, dass der Aspekt des Vertrauens im Schülerinnen und Schüler-Lehrpersonen-Verhältnis für die Offenlegungen sexueller Gewalt nicht ausreichend erforscht

7 Für eine ausführliche Darlegung zur sexuellen Besetzung des weiblichen Oberkörpers im Zusammenhang mit selbst generierten sexuell andeutenden bis expliziten Bildern vgl. Witz 2021. 
ist (vgl. Christmann 2021). Gleichzeitig werden jedoch vertrauensvollen professionellen Beziehungen im pädagogischen Bereich allgemein eine grosse Bedeutung für sowohl Disclosureprozesse (ebenda) als auch für die Thematisierbarkeit sexualitätsbezogener Fragen gegenüber Erwachsenen zugeschrieben. Dabei ist, wie Linke (2020) für Jugendliche im Rahmen von Kinder- und Jugendhilfeeinrichtungen zeigt, für Heranwachsende von Bedeutung, dass sie sich verstanden, ernst genommen und angenommen fühlen (vgl. Linke 2020). Zugleich markieren die Schülerinnen und Schüler eine generationale Grenze zwischen jugendlicher und erwachsener Welt und beschreiben die Offenlegung einer nicht-konsensuellen Praxis gegenüber Personen im Schulsystem als eigentlichen Veröffentlichungsprozess. In der altersbedingten adoleszenten Abgrenzung gegenüber der Erwachsenenwelt, stellt sich aus der Perspektive der Schülerinnen und Schüler die Frage, wie Erwachsene in professionellen Settings vertrauensvolle Ansprechpersonen sein (beziehungsweise bleiben) können und wer im Rahmen von Schule ausserhalb des Bewertungssystems einen intimitätswahrenden Umgang mit der Offenlegung bildbasierter sexueller Gewalterfahrungen bieten kann. Darüber hinaus thematisieren die Schülerinnen und Schüler Scham als Hinderungsgrund sich anzuvertrauen. Dabei zeigt sich zum einen die Abwehr von Scham, indem ein Ansprechen von Veröffentlichungsgeschehen in der ¿Öffentlichkeit) zurückgewiesen wird. Unter Berücksichtigung, dass Scham in der Zeit der frühen Adoleszenz pubertätsbedingt besonders ausgeprägt ist (vgl. Jannink und Witz 2017), kann die Nichtthematisierung jedoch für jene, die direkt oder indirekt am Prozess beteiligt sind, in Teilen auch als soziale Distanzierungsmöglichkeit interpretiert werden, wenn diese der unangenehmen Situation eines Gespräches mit Lehrpersonen ausweichen und damit Interventionsmöglichkeiten Erwachsener sowie Hilfe für Betroffene erschweren. Zum anderen benennen die Schülerinnen und Schüler Scham bei Lehrpersonen, die sie bereits bei der schulischen Bearbeitung sexualitätsbezogener Themen im Unterricht wahrnehmen und welche für sie die Möglichkeit, unangenehme persönliche Dinge zu besprechen, weiter einschränkt. Zwar wird die Reflexion eigener Schamgrenzen auf Seiten von Lehrpersonen als eine Gelingensbedingung für schulischen Sexualkundeunterricht formuliert (vgl. Blumenthal und Damrow 2017, 47), häufig wird Scham in sexualitätsbezogenen Gesprächen jedoch von Fachkräften nicht als doppelte, sich im Verhältnis zu ihrer Zielgruppe (ent-)stehende reflektiert, sondern vornehmlich der Seite der Adressatinnen und Adressaten zugeschrieben (vgl. Jannink und Witz 2017). Auch für Disclosure-Prozesse bei sexueller Gewalt wird Scham auf Seiten der Betroffenen als ein zentraler Hinderungsgrund für Offenlegungen beschrieben (Christmann 2021), jedoch auf Seiten der angesprochenen Fachkräfte kaum beleuchtet. Im Gegensatz dazu wird in der hier analysierten Passage Scham von den Schülerinnen und Schüler auch auf Seiten der Lehrpersonen und somit als doppelte, sich im Verhältnis zu einem Gegenüber entstehende beschrieben. Dabei weisen die Jugendlichen auch darauf hin, dass die Offenbarung 
eigener schambesetzter Erlebnisse eines schamreduzierenden Rahmens bedarf. In der von den Schülerinnen und Schülern beschriebenen Schuldumkehr durch Lehrpersonen zeigt sich schliesslich deren mangelnde Parteilichkeit für Betroffene/Opfer nicht-konsensueller Bildveröffentlichungen sowie das Fehlen einer deutlichen Positionierung zu Fragen des Kinderschutzes (vgl. auch Glammeier 2019, 207). So verweisen die Jugendlichen zugleich auf die fehlende Anerkennung jugendlicher digitaler Lebenswelten sowie mangelnde parteiliche Intervention bei bildbasierten sexuellen Grenzverletzungen und Gewalterlebnissen. Für die Betroffenen/Opfer nicht-konsensueller Bildveröffentlichungen - und insbesondere Mädchen - ergibt sich somit eine doppelte Viktimisierung, da diese aus Sorge für das Versenden von Sexts beschuldigt und bestraft zu werden, Erwachsenen nicht von Problemen in diesem Zusammenhang erzählen. Somit liesse sich sagen, dass sowohl die Furcht vor Victim Blaming als auch Slutshaming, Gewaltsituationen im schulischen Rahmen aufrecht erhalten und den Schaden für die Betroffenen erhöhen können.

\section{6. $\quad$ Fazit}

Der vorliegende Beitrag stellt für den schulischen Kontext Hinderungsgründe für die Offenlegung nicht-konsensueller Praktiken im Umgang mit Sexts heraus. Dabei wird zunächst einmal deutlich, dass Lehrpersonen häufig nichts vom missbräuchlichen Umgang mit intimen Bildern erfahren. Dies führen die Schülerinnen und Schüler auf unterschiedliche Gründe zurück, die zumeist auf Einstellungsfragen und Machtasymmetrien, jedoch auch auf adoleszente und institutionelle Abgrenzungen verweisen. So thematisieren die Schülerinnen und Schüler die Frage eines grundlegenden Vertrauensverhältnisses zu Lehrpersonen, das sie in Bezug auf sexualitätsbezogene Themen und sexuelle Grenzverletzungen als (zusätzlich) eingeschränkt erleben. Auch verweisen sie auf generationale und institutionelle Grenzen, die die Thematisierung auf beiden Seiten erschwert, da Jugendliche die Offenbarung eines Veröffentlichungsgeschehens gegenüber Lehrpersonen als «eigentliche> Veröffentlichung fassen. Zudem benennen sie den Aspekt beidseitiger Scham, sowie Victim Blaming und Slutshaming in Bezug auf Betroffene der Veröffentlichungen, die Seitens Lehrpersonen unreflektiert und unbearbeitet bleiben.

Für die schulische Praxis lassen sich daraus folgende Implikationen diskutieren. Um ein Veröffentlichungsgeschehen im Rahmen von Schule betroffenen-gerecht besprechbar zu machen sowie möglichst schnell zu unterbrechen und damit weitere Viktimisierung zu verhindern, bedarf es zunächst einer Anerkennung einvernehmlichen jugendlichen Sextings als Form digitaler sexueller Kommunikation. Denn während sich in lebensweltorientierten Informationsangeboten für Jugendliche und 
junge Erwachsene ein Normalitätsdiskurs abzeichnet ${ }^{8}$, bleiben Devianz- und Abstinenzdiskurse im Lehrpersonen-Schülerinnen und Schüler-Verhältnis bestehen und beeinflussen negativ die Offenlegung nicht-konsensueller Praktiken. Dabei liesse sich die These formulieren, dass nur (gesellschaftlich) akzeptiertes sexualitätsbezogenes Verhalten auch vertrauensvoll im Generationenverhältnis besprochen werden kann und es Erwachsener bedarf, die - ihre eigenen Themen reflektierend - jugendlichen zugewandt ansprechbar sind. Schulische Prävention und Interventionen sollten bei Veröffentlichungsdynamiken die Beschuldigung von Betroffenen vermeiden und sich darauf konzentrieren, die nicht-einvernehmliche Weitergabe von Sexts zu minimieren. Dabei erfordert es auch die Reflexion geschlechtlicher Zuschreibungen rund um Sexting und Bildveröffentlichungen. Zudem ist ein Bewusstsein für bestehende Machtasymmetrien im Verhältnis von Lehrpersonen und Schülerinnen und Schülern sowie im System Schule bedeutsam. Um «ungünstige Handlungsorientierung» (Glammeier 2019, 206) zu reduzieren, ist die Förderung von Empathie und Perspektivübernahme, wie sie z. B. bei der Prävention von Cybermobbing (lediglich) für Schülerinnen und Schüler formuliert werden (vgl. Schultze-Krumbholz u. a. 2018b), aber auch die Befähigung zu einem schambewussten Umgang mit sexualitätsbezogenen Themen (vgl. Blumenthal und Damrow 2017) auf Seiten von Lehrpersonen relevant. Neben diesen individuellen Anforderungen an schulisches Personal ist für alle Akteurinnen und Akteure die Benennung von Zuständigkeiten («Wer gibt wann, was weiter?») sowie die Klärung von Kompetenzen, Handlungsabläufen ${ }^{9}$ und die Kooperationen mit Fachberatungsstellen, wie sie im Rahmen von institutionellen Schutzkonzepten ausgearbeitet werden, nicht nur wünschenswert sondern dringend erforderlich.

8 Vgl. hierfür z. B. die Youtube-Filme «Nacktbilder versenden? Gut? Schlecht? SEXTING» des Channels Jungsfragen, https://www.youtube.com/watch?v=-TYGmIVE6OA oder «Wenn die ganze Schule dein NACKTBILD sieht!» von Auf Klo, https://www.youtube.com/watch?v=5bligm593xy.

9 Vgl. z. B. «Erste Schritte für Pädagogen und Lehrkräfte». https://www.klicksafe.de/themen/problematische-inhalte/sexting/was-tun-wenns-schief-geht-handlungsempfehlungen/. 


\section{Literatur}

Blumenthal, Sara-Friederike, und Miriam Damrow. 2017. «Blumenthal, Sara-Friederike; Damrow, Miriam: Tabus in der Sexualaufklärung. Wie Scham das Sprechen über Sexualität prägt.» Erziehung \& Unterricht, 167(1-2), 44-50.

Böhm, Maika, Jürgen Budde, und Arne Dekker. 2018. «Sexuelle Grenzverletzungen mittels digitaler Medien an Schulen - Annäherung an einen doppelten Verdeckungszusammenhang». MedienPädagogik: Zeitschrift für Theorie und Praxis der Medienbildung 2018 (Occasional Papers): 1-18. https://doi.org/10.21240/mpaed/00/2018.02.21.X.

Bohnsack, Ralf. 2013. «Typenbildung, Generalisierung und komparative Analyse: Grundprinzipien der dokumentarischen Methode». In Die dokumentarische Methode und ihre Forschungspraxis: Grundlagen qualitativer Sozialforschung, herausgegeben von Ralf Bohnsack, Iris Nentwig-Gesemann, und Arnd-Michael Nohl, 241-70. Wiesbaden: VS Verlag für Sozialwissenschaften. https://doi.org/10.1007/978-3-531-19895-8_11.

«Bund und Länder gemeinsam gegen sexuelle Gewalt an Schulen». o. J. «Bund und Länder gemeinsam gegen sexuelle Gewalt an Schulen - UBSKM». https://beauftragter-missbrauch. de/presse/pressemitteilungen/detail/bund-und-laender-gemeinsam-gegen-sexuelle-gewalt-an-schulen.

Christmann, Bernd. 2021. Disclosure von sexualisierter Gewalt in schulischen Kontexten: Fachkräfte als Ansprechpersonen betroffener Schüler*innen. Sexuelle Gewalt und Pädagogik. Wiesbaden: VS Verlag für Sozialwissenschaften. https://doi.org/10.1007/978-3-658-331429.

Dekker, Arne, Thula Koops, und Peer Briken. 2016. Sexualisierte Grenzverletzungen und Gewalt mittels digitaler Medien. Zur Bedeutung digitaler Medien für Phänomene sexualisierter Grenzverletzungen und Gewalt gegen Kinder und Jugendliche. UBSKM.

Döring, Nicola. 2012. «Erotischer Fotoaustausch unter Jugendlichen: Verbreitung, Funktionen und Folgen des Sexting». Zeitschrift für Sexualforschung 25 (1): 4-25. https://doi. org/10.1055/s-0031-1283941

Döring, Nicola. 2015. «Sexting. Aktueller Forschungsstand und Schlussfolgerungen für die Praxis». In Gewalt im Netz: Sexting, Cybermobbing \& Co, 15-43. Blickpunkt Kinder- und Jugendschutz. Berlin: Bundesarbeitsgemeinschaft Kinder- und Jugendschutz. Zugegriffen 8. Juni 2021. http://www.nicola-doering.de/wp-content/uploads/2014/08/D\%C3\%B6ring2015-Sexting.-Aktueller-Forschungsstand-und-Schlussfolgerungen-f\%C3\%BCr-die-Praxis. pdf.

Eichenberg, Christiane und Felicitas Auersperg. 2018. Chancen und Risiken digitaler Medien für Kinder und Jugendliche: ein Ratgeber für Eltern und Pädagogen. 1. Aufl. Göttingen: Hogrefe. http://doi.org/10.1026/02647-000

Glammeier, Sandra. 2019. "Sexuelle Gewalt und Schule». In Sexuelle Gewalt in pädagogischen Kontexten, herausgegeben von Martin Wazlawik, Heinz-Jürgen Voß, Alexandra Retkowski, Anja Henningsen, und Arne Dekker, 3:197-209. Sexuelle Gewalt und Pädagogik. Wiesbaden: Springer Fachmedien Wiesbaden. https://doi.org/10.1007/978-3-658-18001-0_14. 
Hofherr, Stefan. 2019. «Klassen der Offenlegung sexueller Gewalt». In Sexuelle Gewalt in pädagogischen Kontexten: Aktuelle Forschungen und Reflexionen, herausgegeben von Martin Wazlawik, Heinz-Jürgen Voß, Alexandra Retkowski, Anja Henningsen, und Arne Dekker, 137-49. Sexuelle Gewalt und Pädagogik. Wiesbaden: Springer Fachmedien. https://doi. org/10.1007/978-3-658-18001-0_10.

Jannink, Helge, und Christina Witz. 2017. «Die Doppelseitigkeit der Scham in der sexualpädagogischen Arbeit mit jugendlichen Geflüchteten». In Sexualität und Gender im Einwanderungsland: öffentliche und zivilgesellschaftliche Aufgaben - ein Lehr- und Praxishandbuch, herausgegeben von Uwe Sielert, Helga Marburger, und Christine Griese, 127-39. Berlin/ Boston: De Gruyter Oldenbourg. https://doi.org/10.1515/9783110518351-008.

Johnson, Matthew, Faye Mishna, Moses Okumu, und Joanne Daciuk. 2018. Non-Consensual Sharing of Sexts: Behaviours and Attitudes of Canadian Youth. Ottawa: MediaSmarts. https://mediasmarts.ca/sites/mediasmarts/files/publication-report/full/sharing-of-sexts. pdf.

Linke, Torsten. 2020. Sexuelle Bildung in der Kinder- und Jugendhilfe: die Bedeutung von Vertrauenskonzepten Jugendlicher für das Sprechen über Sexualität in pädagogischen Kontexten. Angewandte Sexualwissenschaft. Gießen: Psychosozial-Verlag. https://doi. org/10.30820/9783837976816.

Madigan, Sheri, Anh Ly, Christina L. Rash, Joris Van Ouytsel, und Jeff R. Temple. 2018. «Prevalence of Multiple Forms of Sexting Behavior Among Youth: A Systematic Review and Meta-Analysis». JAMA Pediatrics 172 (4): 327-35. https://doi.org/10.1001/jamapediatrics.2017.5314.

Naezer, Marijke, und Lotte Oosterhout. 2020. «Only sluts love sexting: youth, sexual norms and non-consensual sharing of digital sexual images». Journal of Gender Studies 30 (1): 79-90. https://doi.org/10.1080/09589236.2020.1799767.

Nentwig-Gesemann, Iris. 2013. «Die Typenbildung der dokumentarischen Methode». In Die dokumentarische Methode und ihre Forschungspraxis: Grundlagen qualitativer Sozialforschung, herausgegeben von Ralf Bohnsack, Iris Nentwig-Gesemann, und Arnd-Michael Nohl, 295-323. Wiesbaden: VS Verlag für Sozialwissenschaften. https://doi.org/10.1007/978-3531-19895-8_13.

Oeffling, Yvonne, Veronika Winter, und Mechthild Wolff. 2018. «Prävention als organisationales Bildungskonzept». In Lehrbuch Schutzkonzepte in pädagogischen Organisationen: Mit Online-Materialien, herausgegeben von Carolin Oppermann, Veronika Winter, Claudia Harder, Mechthild Wolff, und Wolfgang Schröer, 1. Auflage, 204-231. Studienmodule Soziale Arbeit. Weinheim Basel: Beltz Juventa.

Przyborski, Aglaja. 2004. Gesprächsanalyse und dokumentarische Methode: qualitative Auswertung von Gesprächen, Gruppendiskussionen und anderen Diskursen. 1. Auflage. Lehrbuch. Wiesbaden: Springer Fachmedien Wiesbaden GmbH.

Schneider, Hans Joachim. 2014. Kriminologie: ein internationales Handbuch. Band 1: Grundlagen. DeGruyterHandbuch. Berlin [u.a.]: DeGruyter. https://doi.org/10.1515/9783110248272. 
Schultze-Krumbholz, Anja, Markus Hess, Jan Pfetsch, und Herbert Scheithauer. 2018. "Who is involved in cyberbullying? Latent class analysis of cyberbullying roles and their associations with aggression, self-esteem, and empathy». Cyberpsychology: Journal of Psychosocial Research on Cyberspace 12 (4). https://doi.org/10.5817/CP2018-4-2.

Schultze-Krumbholz, Anja, Pavle Zagorscak, Anne Roosen-Runge, Herbert Scheithauer, und Ernst Reinhardt Verlag. 2018. Medienhelden: Unterrichtsmanual zur Förderung von Medienkompetenz und Prävention von Cybermobbing. 2. Auflage. München: Ernst Reinhardt Verlag.

«Sexuelle Gewalt mittels digitaler Medien - UBSKM». o. J. https://beauftragter-missbrauch.de/ praevention/sexuelle-gewalt-mittels-digitaler-medien.

Strasburger, Victor C., Harry Zimmerman, Jeff R. Temple, und Sheri Madigan. 2019. «Teenagers, Sexting, and the Law». Pediatrics 143 (5): e20183183. https://doi.org/10.1542/peds.20183183.

Symons, Katrien, Koen Ponnet, Michel Walrave, und Wannes Heirman. 2018. «Sexting Scripts in Adolescent Relationships: Is Sexting Becoming the Norm?» New Media \& Society 20 (10): 3836-57. https://doi.org/10.1177/1461444818761869.

Van Ouytsel, Joris, Ellen Van Gool, Michel Walrave, Koen Ponnet, und Emilie Peeters. 2016. «Sexting: Adolescents' Perceptions of the Applications Used for, Motives for, and Consequences of Sexting». Journal of Youth Studies, November, 1-25. https://doi.org/10.1080/1 3676261.2016.1241865.

Vogelsang, Verena. 2017. Sexuelle Viktimisierung, Pornografie und Sexting im Jugendalter: Ausdifferenzierung einer sexualbezogenen Medienkompetenz. Medienbildung und Gesellschaft. Wiesbaden: Springer VS. https://doi.org/10.1007/978-3-658-16843-8.

Witz, Christina. 2021. «Sexting | Körper-Bilder | Geschlecht. Orientierungen Jugendlicher auf sexuell interpretierbare visuelle Selbstdarstellungen». GENDER - Zeitschrift für Geschlecht, Kultur und Gesellschaft 13 (3): 106-21. https://doi.org/10.3224/gender.v13i3.08. 